\section{Berufsverband der Deutschen Urologen e.V.}

Urologe 2009.48: 328

DOI 10.1007/s00120-009-1958-8

๑) Springer Medizin Verlag 2009

\section{Redaktion}

H. Jonitz, Darmstadt

\title{
++* URO-Telegramm
}

Im Streit um die Honorarreform für Ärzte wirft NRW-Gesundheitsminister Karl-Josef Laumann (CDU) der Kassenärztlichen Bundesvereinigung Versagen vor. Wir haben es mit einem nicht mehr nachvollziehbaren System zu tun", sagte Laumann der „Rheinischen Post". Das System sei "völlig intransparent".

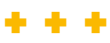

Der Zulassungsausschuss in Schleswig-Holstein hat Anträge von 107 Augenärzten auf ein zeitlich befristetes Ruhen der Zulassung abgelehnt.Die Anträge wurden mit dem Votum der Kassen formal zurückgewiesen, da die Begründung nicht individuell genug gewesen sei, berichtet Dr. Bernhard Bambas, Landesvorsitzender Schleswig-Holstein des Berufsverbandes der Augenärzte (BVA) Außerdem habe es sich um ein abgestimmtes Vorgehen der Augenärzte gehandelt.!

$$
+4+4
$$

\section{Die Öffnung der Krankenhäuser} für die spezialisierte ambulante Versorgung bleibt in Niedersachsen umstritten. Die Kassenärztliche Vereinigung Niedersachsen (KVN) hat eigenen Angaben nach bisher sieben Klagen gegen die Genehmigungen des Niedersächsischen Ministeriums für Soziales, Frauen, Familie und Gesundheit zur Öffnung der Krankenhäuser vor den Sozialgerichten angestrengt. Diese Klagen haben aufschiebende Wirkung, so dass die Genehmigungen ausgesetzt wurden.
„Wir sehen uns nicht mehr in der Lage, den Spagat zwischen medizinisch notwendiger Diagnostik und Therapie und dem viel zu geringen Honorar, das uns die Kassen zur Verfügung stellen, zu machen", schreiben die Stuttgarter HNO-Ärzte in einer Pressemitteilung. „Das Honorarbudget für die umfassende HNO-Basisversorgung muss zunächst mindestens 58 Euro pro Fall betragen", fordern die Mediziner.

$$
4+4
$$

Nach Meinung des Bundesverbandes der Deutschen Industrie (BDI) muss das Projekt der elektronischen Gesundheitskarte unverzüglich starten: „Der intelligente Einsatz von Kommunikationstechnologien im Gesundheitswesen bringt einen enormen Schub in der medizinischen Versorgung. Davon profitieren die Patienten. Zugleich gibt es kräftige Impulse für Wachstum und Beschäftigung" erklärte BDI-Hauptgeschäftsführer Werner Schnappauf.

$$
+4+
$$

Die Kassenärztliche Bundesvereinigung hat die Rüge der BKK wegen ihres Schreibens an die Niedergelassenen zur Diagnosecodierung begrüßt., Es ist richtig und wichtig, dass das Bundesversicherungsamt durchgegriffen hat", sagte Vorsitzender Dr. Andreas Köhler in Berlin. Diagnosen dürften sich nicht nach wirtschaftlichen Begehrlichkeiten richten!
Die Kassenärztliche Vereinigung (KV) Berlin hat ein Behandlungsprogramm für Patienten mit akuten Rückenschmerzen im Lendenwirbelbereich nach § 73c SGB V mit der Kaufmännischen Krankenkasse (KKH) geschlossen. Anfang März starte das Programm, teilten KV und Kasse in Berlin mit.

$$
\text { 나 내 나 }
$$

gespannt, „, die gegenwärtige Wirtschaftskrise wird aber dazu führen, dass sich viele Kommunen ab 2010 und 2011 von ihren Kliniken trennen werden", prophezeit Fresenius-Chef Ulf Schneider.

$$
+4+
$$

Die Techniker Krankenkasse hat nach eigenen Angaben seit Einführung des Gesundheitsfonds 23.000 neue Mit-

Weil er nach Nichtbegleichung seiner Liquidation einer Patientin an deren Wohnungstür einfach die Prothesen aus dem Mund entfernt hat, muss ein 57-jähriger Zahnarzt aus Neu-Ulm 6.000 Euro Geldstrafe zahlen. Das Amtsgericht Neu-Ulm verurteilte den Arzt am Freitag wegen Nötigung und vorsätzlicher Körperverletzung.

$$
\text { yㅐ }
$$

Der Gesundheitskonzern Fresenius aus Bad Homburg erwartet durch die Finanzkrise eine neue Privatisierungswelle bei den kommunalen Krankenhäusern. Schon jetzt seien die städtischen Haushalte stark anglieder gewonnen. „Seitdem der Beitragssatz aller Krankenkassen gleich hoch ist, gewinnen wir Mitglieder in Regionen, in denen Konkurrenten einen niedrigeren Beitragssatz hatten", sagte der Vorstandschef Norbert Klusen der "Leipziger Volkszeitung"

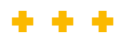

Der Basistarif in der privaten Krankenversicherung hat schlechte Noten von der Stiftung Warentest erhalten. Auch werde der Tarif kaum in Anspruch genommen, berichtet die „Frankfurter Rundschau“.

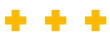

Anlässlich des 55. Kongresses der Nordrhein-Westfälischen Gesellschaft für Urologie vom 30. April bis 02. Mai 2009 in der Halle Münsterland in Münster/Westfalen, findet am Samstag, den 02. Mai um 09.00 Uhr ein hochinteressanter

\section{„Netzwerk - Workshop"}

für Urologinnen und Urologen in Netzen und solchen, die es werden wollen, statt.

Die verschiedenen Workshop - Themen beinhalten beispielsweise „Motivations-Schaffung ${ }^{\prime \prime}$, Professionelle Organisationsstrukturen in Netzen“, ,'Gewinnerzielung in Netzen",,'Versorgungsforschung und Sicherung der Uro-Onkologischen Kompetenz durch Zentrenbildung ", ${ }^{\prime \prime}$, Verbindliches Handeln in Netzen ",, Professionelle Außendarstellung Urologischer Netze" sowie „Round-Table-Diskussionen".

Jeder, der sich für moderne Kooperationsformen oder für "Netz-Arbeit" interessiert, ist herzlich zu dieser Veranstaltung eingeladen, ebenso auch Kolleginnen und Kollegen, die dem Netz-Gedanken noch distanziert gegenüberstehen und sich einfach nur informieren wollen.

\section{Ort: Halle Münsterland}

Zeit: Samstag, 02. Mai 2009, 09 Uhr.

Auskunft:www.nrwgu.de, www.urologie-nrwgu.de.

Mail:nrwgu2009@klinikum-os.de 